\title{
Proposta de Vim On-Demand para fatiamento de Nuvem
}

\author{
Eduardo H. R. Zanela ${ }^{1}$, Cesar A. C. Marcondes ${ }^{2}$, Fábio L. Verdi ${ }^{1}$ \\ Alex M. G. de Almeida ${ }^{3}$, Emerson R. A. Barea ${ }^{1}$ \\ ${ }^{1}$ Departamento de Computação - UFSCar \\ ${ }^{2}$ Instituto Tecnológico da Aeronáutica - ITA \\ ${ }^{3}$ Faculdade de Tecnologia de Ourinhos - FATEC \\ eduardo-hnq@hotmail.com \\ \{marcondes, verdi, emerson.barea\} @ufscar.br \\ alex.marino@fatecourinhos.edu.br
}

\begin{abstract}
This paper presents a proposal for a Virtualized Infrastructure Manager On-Demand for cloud slicing that allows bare metal control and network services with focus in on-demand creation. For this, a management system was implemented using Openstack in conjunction with virtualization tools allowing the instantiation of a physical machine. In this paper, we present 3 options of installation: complete instantiation bare metal, complete instantiation with containers and complete instantiation with preloaded image. Our results indicate that the proposed, implemented, orchestrated and tested architecture meets the requirements of VIM On-Demand, and points out the main prevailing points in VIM.
\end{abstract}

Resumo. Este trabalho apresenta uma proposta de VIM Sob-Demanda para fatiamento de nuvens que permita controle bare metal e de serviços de redes, com enfoque na sua criação sob demanda. Para isto, foi implementado um sistema de gerenciamento utilizando o Openstack em conjunto com ferramentas de virtualização, permitindo a instanciação de uma máquina física. Neste artigo, apresentamos 3 opções de instalação: instanciação completa bare metal, instanciação completa com containers e instanciação completa com imagem pré-carregada. Nossos resultados indicam que a arquitetura proposta, implementada, orquestrada e testada atende os requisitos do VIM On-Demand, e apontam os principais pontos preponderantes no VIM.

\section{Introdução}

Um dos maiores desafios enfrentados no desenvolvimento da rede $5 \mathrm{G}$ é atender às diversas demandas de consumidores por novos serviços, modificando a forma de implantação de redes de tamanhos fixos para tamanhos variáveis, possibilitando atender aos requisitos de desempenho específicos quanto à latência, escalabilidade, disponibilidade e confiabilidade, impostos por cada caso de uso.

Para tal, é necessária a introdução do fatiamento de rede baseado em demandas, que permitirá que cada fatia de rede, denominado como slice, seja alocada conforme a demanda e requisitos do locatário em função de suas aplicações. O ponto central da 
implantação do fatiamento é dependente da orquestração de atividades que possibilitem a instalação, preparação do ambiente sucedida de suas configurações e instalação dos serviços requeridos. A conjuminação destas tarefas visa, por fim, a elaboração de um aparato de gerenciamento de fatias de redes virtualizadas, as quais adotam anglicismo Virtualized Infrastructure Manager (VIM).

A definição de slice de rede pode ser dado como redes lógicas executadas em rede física ou virtual, mutuamente isoladas, dotadas de controle e gerenciamento independentes, que podem ser criados sob demanda [Clayman 2017].

Uma das alternativas que vem sendo utilizada para demonstração do conceito apresentado é a virtualização da rede, que possibilita transformar redes reais em redes virtuais, utilizando soluções tecnológicas baseadas, principalmente, em software tais como: Software Defined Networking (SDN), Network Functions Virtualization (NFV) e Virtualized Network Function (VNF). Estas tecnologias concedem capacidade programática à solução, flexibilidade e modularidade para o desenvolvimento de diferentes redes virtuais para diferentes casos de uso.

Dentre os aspectos importantes correlacionados ao desenvolvimento do VIM, são as verificações dos pontos preponderantes relativos à escalabilidade, aferições pertinentes à resiliência do ambiente e constatações dos tempos gastos durante o processamento de cada etapa da instalação. A proposta apresentada neste trabalho aponta para o desafio de um VIM de fatiamento de rede que criem slices sob demanda. Espera-se que os slices caracterizem leveza, praticidade, facilidade de instalação e configuração, somando-se ainda à capacidade de gerenciamento dos componentes de infraestruturas virtualizadas. Ao término deste trabalho, espera-se responder duas questões: (i) Os recursos computacionais, tais como memória principal e quantidade de núcleos são determinantes para a eficiência do processo? (ii) É possível aferir vantagem em desempenho da abordagem VIM on-demand em relação à tradicional?

No melhor dos nossos esforços, este trabalho apresenta uma proposta de desenvolvimento de um VIM sob demanda (VIM on-Demand), no qual será apresentado o desenvolvimento de forma virtualizada e dividido em 3 opções de instalações: Instanciação Completa Bare Metal; Instanciação Completa com Containers e Instanciação Completa com Imagem Pré-Carregada. Atribui-se como aspecto inovador desta proposta (i) o uso de equipamentos de baixo custo, (ii) uso exclusivo de tecnologias de código aberto, (iii) orquestração total da implementação e (iv) instanciação de diferentes casos de uso. Ressalta-se a escolha da utilização do Openstack em função dos seus serviços aderentes ao propósito deste trabalho, que são a existência do bare metal e serviços de controle de computação.

O restante desse trabalho está organizado da seguinte maneira: a Seção 2 relaciona os trabalhos correlatos a este. Na Seção 3 são apresentados os principais conceitos envolvidos no trabalho, o aparato tecnológico e a metodologia empregada na execução dos experimento. Na Seção 4 são discutidos os resultados obtidos no rol de experimentos realizados e, por fim, na Seção 5 conclui este trabalho e apresenta sugestões de trabalhos futuros. 


\section{Trabalhos Relacionados}

Apesar do conceito de 5G ser considerado novo, existem diversos trabalhos acadêmicos que colaboram com o seu desenvolvimento, porém o único que utiliza o conceito de Slice as a Service, fator central para o provisionamento VIM on-demand, é o projeto NECOS ${ }^{1}$, no qual este trabalho faz parte [Silva et al. 2018].

Como outros projetos e trabalhos acadêmicos, destacam o 3GPP, o qual apresentou a proposta de padronização do sistema de rede de acesso $5 \mathrm{G}$ e do núcleo de sistema de rede 5G ao mesmo tempo [Kim et al. 2017]; o 5G NORMA, que propôs-se uma arquitetura capaz de lidar eficientemente com diversos requisitos e flutuações na procura de tráfego resultantes de conjunto de serviços heterogêneos e suspensos [Rost et al. 2017]; e o SONATA abordou os desafios significativos associados ao desenvolvimento e à implantação dos serviços complexos previstos para as redes 5G [Dräxler et al. 2017].

O trabalho [Freitas et al. 2018], apresenta um sistema capaz de implantar e fornecer VIMs operacionais completos através de um Data Center (DC) slice Controller, com base em uma arquitetura em que as fatias do DC são criadas sobre recursos transformáveis (computação e armazenamento). O presente trabalho tem como diferenças do trabalho de [Freitas et al. 2018], a instalação total do ambiente de controle sob demanda analisando diferentes abordagens para carregamento do ambiente. Além disso, entende-se que ao realizar a instalação do ambiente ao invés de importar imagens prontas, mais flexibilidade e opções de customização de tal ambiente podem ser exploradas.

Existem diversos trabalhos voltados à indústria, e para o presente trabalho foram analisados o Kayobe ${ }^{2}$, que é um projeto Openstack com finalidade de automatizar a implantação do Openstack em um conjunto de servidores bare metal, utilizando containers; e o TripleO (Openstack On Openstack) ${ }^{3}$ que é um projeto do Openstack que permite implantar duas nuvens, com a finalidade de instalar, atualizar e operar nuvens Openstack utilizando as próprias instalações de nuvem do Openstack como base.

Em relação à característica inovadora nessa criação de slice, comparado com a utilização de recursos no OpenStack Nova, compreende-se que o mesmo permite assimilar uma mini-nuvem para o locatário.

\section{Materiais e Métodos}

Nesta seção tem-se descritos os materiais e métodos utilizados durante o desenvolvimento da proposta; na Subseção 3.1 são apresentados os principais conceitos e definições envolvidos neste trabalho; na Subseção 3.3 o aparato computacional e tecnológico utilizado para realização deste trabalho; na Subseção 3.2 os procedimentos adotados que possibilitaram perfazer as atividades e, por fim, na Subseção 3.4 apresentamos a descrição dos experimentos.

\subsection{Conceitos e Definições}

Para efetivar o desenvolvimento da proposta de VIM on-demand nas condições propostas, alguns requisitos devem ser previamente elencados e discutidos. Os requisitos que

\footnotetext{
${ }^{1}$ http://www.h2020-necos.eu/.

${ }^{2}$ https://kayobe.readthedocs.io.

${ }^{3}$ https://wiki.openstack.org/wiki/TripleO.
} 
nortearam o processo de implementação, configuração e instanciação do ambiente e experimentos, seguem descritos:

\subsubsection{Virtualização}

Permite criar uma versão similar de algo, um software adaptável como sistemas operacionais, dispositivos, hardware ou recursos de redes. A computação em nuvem, faz intensa utilização do conceito da virtualização, uma vez que a mesma proporciona uma redução de custo e melhor controle de seu ambiente, segregando a aplicação e sistema operacional dos componentes físicos de origem.

A virtualização dos serviços de rede implica na transformação de suas funções de redes estáticas em funções virtuais. Para [Han et al. 2015], a virtualização das funções de redes propõem melhorar o provisionamento dos serviços de redes. A transformação de redes reais em redes virtuais pressupõe a aplicação de tecnologias baseadas em software, conferindo capacidade de controle, flexibilidade e programação, além de colaborar com o desenvolvimento de diferentes redes virtuais para diferentes casos de uso.

\subsubsection{Fatiamento de Rede}

Trata-se de uma técnica específica de virtualização, a qual proporciona a implementação de várias redes lógicas sob uma infraestrutura de rede física compartilhada [Mademann 2017]. As redes lógicas dos slices atuam de ponta a ponta e são formadas por funções de rede, armazenamento e computação [Freitas et al. 2018].

No fatiamento, a infraestrutura de uma rede é subdividida em partes denominadas slices. A slice é reservada à uma aplicação de um usuário ou locatário e é considerada como um conjunto de recursos que, combinados com a orquestração, atendem a todos os requisitos de um caso de uso específico [Clayman et al. 2018].

A segregação da infraestrutura em múltiplas partes confere benefícios econômicos, tais como: redução de gastos, remoção das limitações de infraestrutura e tecnologias, expansão do cenário para vários clientes diferentes e menor transmissão de dados na rede, isto porque o tráfego de dados móveis é crescente [Silva et al. 2018].

Um caso de uso que tem como alvo a utilização de nuvens para oferecer serviços e infraestrutura para propósito geral, é composto por três tipos de agentes:

- Provedor de infraestrutura: é o provedor que possui e gerencia uma determinada rede física e recursos computacionais, armazenamento e comunicação;

- Locatário: aluga os serviços de infraestrutura de rede de um ou mais provedores, sob a forma de rede virtual;

- Usuário final: aluga os serviços tanto de infraestrutura de redes ofertados pelos provedores, quanto os serviços fornecidos pelos locatários, porém não consegue fornecer recurso para outros usuários.

Nos slices, os recursos são unidades gerenciáveis e são divididos em VNFs e recursos de infraestrutura. Para cada slice é necessário que exista um VIM gestor do controle dos recursos, suportando diferentes demandas, denominado como VIM on-Demand [Silva et al. 2018]. 


\subsubsection{Slice as a Service}

Refere-se a um novo conceito de serviço de rede, cujos recursos são acoplados internamente à slice e gerenciados como um todo, podendo ainda conter componentes de serviços independentes de outros slices [Ordonez-Lucena et al. 2017].

O slice deve ser criada diretamente em ambiente de nuvens, o que proporcionará menor tempo de resposta nas alterações das demandas de serviços. O Slice as a Service possui o conceito de princípio leve, o qual exaure ao limite as configurações dos slices, recursos físicos e capacidade de gerenciamento de recursos e desempenho por software.

Em uma situação onde os recursos de infraestrutura ficam inutilizados devido ao excesso de recursos, o conceito do Slice as a Service torna possível eliminar a ociosidade da rede, criando novos slices conforme requisitados pelos clientes, proporcionando diferentes VIMs e funções virtualizadas de rede. Para [Freitas et al. 2018], os slices permitem uma visão geral dos mecanismos, componentes, e abstrações que podem ser utilizados para fornecer um modelo de Slice as a Service baseado na instanciação VIM dinâmica.

O problema da alocação dos slices possui um forte componente de otimização. Em um sistema com escala maior e com caminhos de rede disjuntos (Figura 1), a escolha de enlaces para serem alocados para cada slice pode definir a quantidade de clientes suportados pela rede, bem como o desempenho da rede para tais clientes. O VIM é um dos principais componentes de uma estrutura virtualizada, cabe-lhe a responsabilidade de manter o controle e gerenciamento dos recursos de computação, armazenamento e infraestrutura de rede [Freitas et al. 2018].

Oposto às tradicionais configurações de um único VIM para toda infraestrutura da computação em nuvem, o VIM on-Demand é implantado dinamicamente para cada slice, cabendo a ele permitir que a slice de rede esteja apta ao atendimento de diferentes casos de uso, independentemente da necessidade de execução de qualquer tipo de tecnologia.

\subsection{Procedimentos e Técnicas}

Nesta subseção será apresentado um conjunto de tarefas necessários à implantação de um VIM on-Demand. A Figura 1 permite visualizar este conjunto de tarefas e suas dependências entre si:

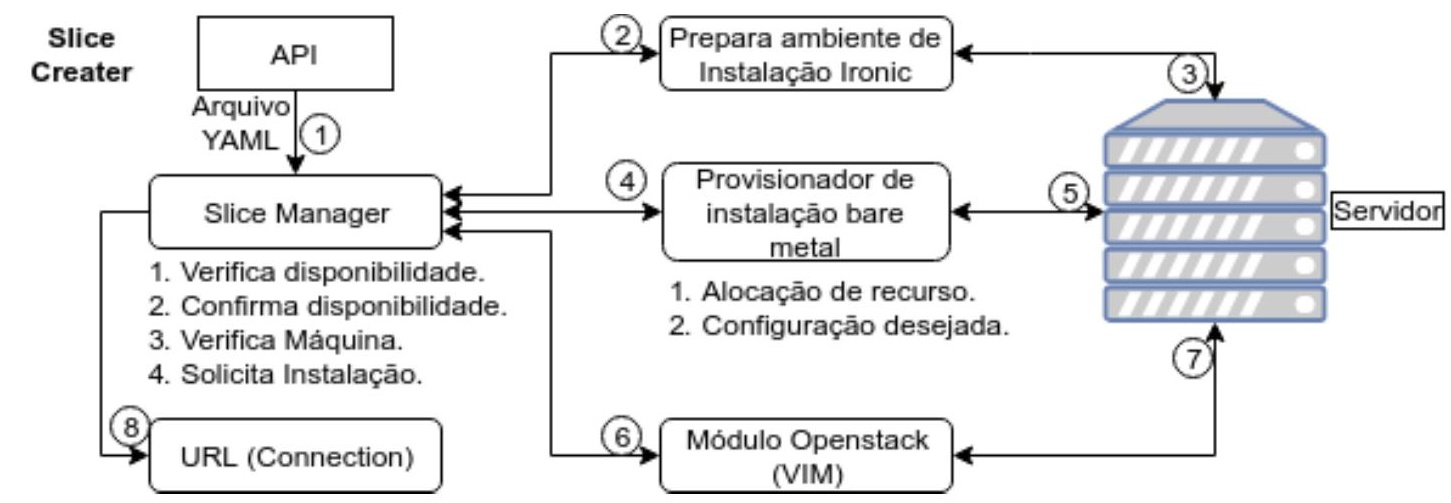

Figura 1. Infraestrutura VIM On-Demand.

A seguir serão apresentadas três opções de instalação VIM on-Demand proposta 



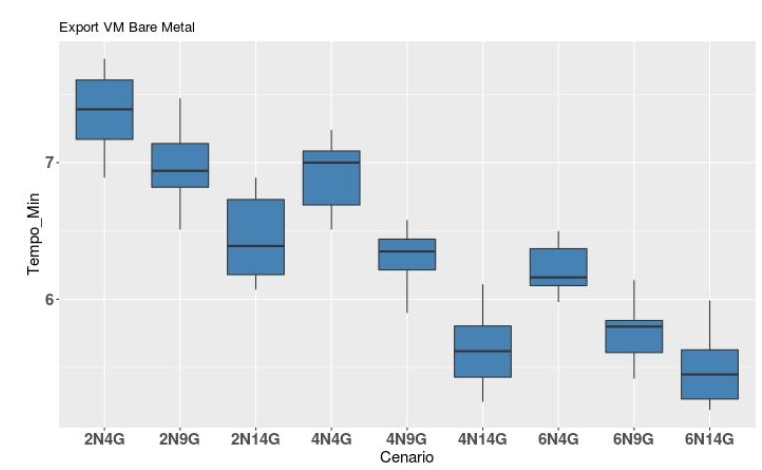

(a) Export VM Bare Metal (ExpVMBM)

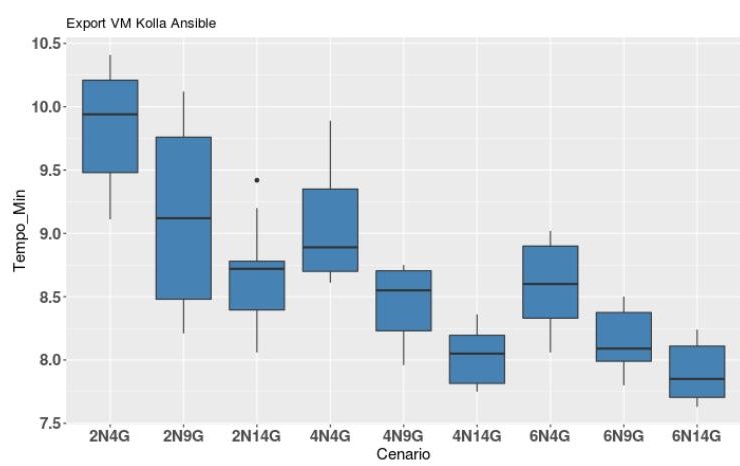

(c) Export VM Kolla-Ansible (ExpVMKA)

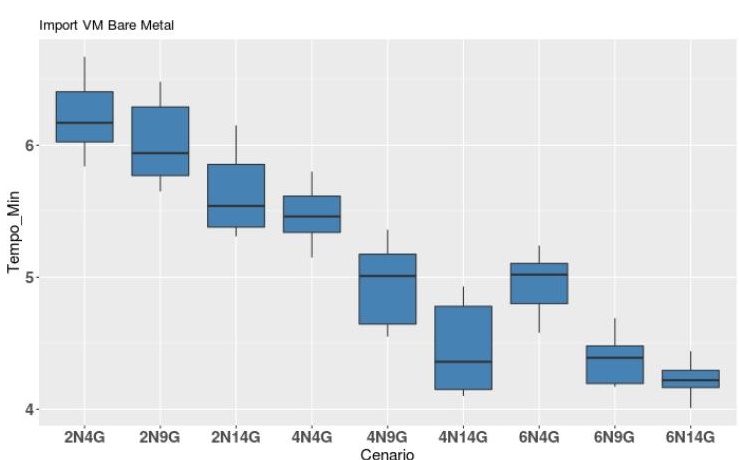

(b) Import VM Bare Metal (ImpVMBM)

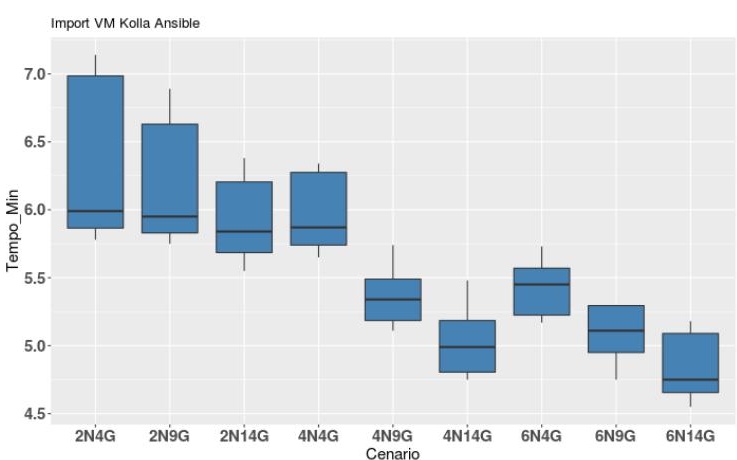

(d) Import VM Kolla-Ansible (ImpVMKA)

Figura 7. Exportação e importação de máquinas virtuais.

Corroborando para a constatação da prevalência da quantidade de memória em detrimento do número de núcleos, notamos nas Figuras 8 a e $8 b$, uma ligeira superioridade quanto a estabilidade, ou seja, as barras relativas às configurações com 14 gigabytes de memória sobre as configurações com 6 núcleos.

A Figura 8a apresenta os maiores tempos para cada configuração de máquina virtual durante o processo de execução dos testes $\boldsymbol{B M}, \boldsymbol{K A}$ e $\boldsymbol{O S}$, enquanto a Figura 8b, ao contrário da anterior, apresenta os menores tempos durante o processo de execução dos testes. Ambas apresentam a variação de período durante a execução para cada cenário, permitindo visualizar, embora timidamente, uma relevante diferença entre cada cenário de configurações das máquinas virtuais.

Considerando o comportamento homogêneo para os cenários apresentados até o momento é possível afirmar à questão $(i)$ através dos recursos computacionais que são preponderantes na eficiência do processo e dadas nossas observações a memória principal, prevalece sobre a quantidade de núcleos.

Com relação às médias das tomadas de tempo para os experimentos, a Figura 6d exibe as médias e respectivas marcações de desvio padrão. É possível observar que obstante a onerosidade do tempo de instalação do bare metal (Figura 6a), os tempos dos passos de Exportação e Importação de VMs (Figura 7) são extremamente vantajosos em relação aos tempos de instalação do Kolla-Ansible (Figura 6b) e Openstack (Figura 6c).

É notória a diferença estatística das atividades de não instalação, ou seja, importa- 


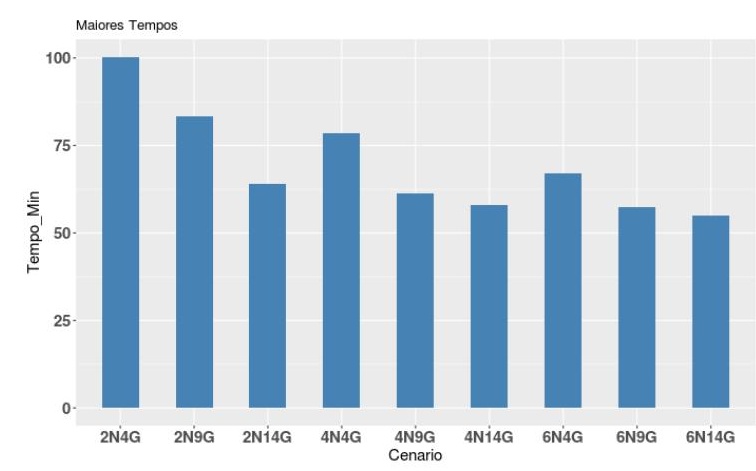

(a) Maiores Tempos

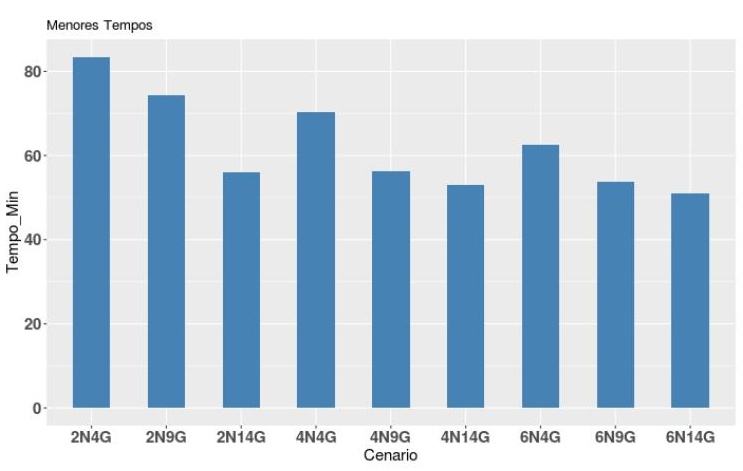

(b) Menores Tempos

Figura 8. Tempo MAX e MIN

ção de configurações de VMs sobre os tempos de instalação, pois os tempos das mesmas são inferiores ao tempo médio de ambas e subtraídas do desvio padrão. Deste modo, pode-se afirmar que a resposta para a questão (ii) aponta no sentido de que a abordagem VIM on-demad é fortemente vantajosa, implicando na eficiência de utilização de recursos computacionais sob esta abordagem.

\section{Considerações finais}

Este trabalho apresentou um modelo de VIM on-demand, a qual possibilita instanciar máquinas a nível bare metal e serviços de computação, proporcionando, ao final, o seu controle e gerenciamento. Além disso, também foi possivel dividir o processo de instalação, configuração e instanciação em 3 etapas e exportar um snapshot para cada ambiente: (I) Instanciação Completa Bare Metal, (II) Instanciação Completa com Containers e (III) Instanciação Completa com Imagem Pré-Carregada.

Ao concluir os experimentos, observa-se que os melhores resultados durante os experimentos foram os que usaram memória RAM com 14 GB (G14), a maior quantidade de memória utilizada durante os testes. Diante disso, conclui-se que a quantidade de memória é aspecto preponderante no VIM on-Demand, pois os valores que apresentaram melhor desempenho foram em situações de maior quantidade de memória, e não alteração de outros fatores como quantidade de núcleos. Adicionalmente à preponderância da quantidade de memória, foi possível constatar que a abordagem VIM on-demand confere ganho computacional no tocante a eficiência de uso dos recursos.

Como trabalhos futuros, pretende-se incluir outros controladores, como o VLSP e Kubernets, no VIM on-Demand, e realizar novos experimentos analisando consumo de energia, desempenho de memória e processamento com casos de usos que necessitem de uma sobrecarga.

\section{Agradecimentos}

Esta pesquisa é financiada pela Comissão Europeia e Ministério da Ciência, Tecnologia, Inovação e Comunicação (MCTIC) através da RNP e CTIC no contexto da $4^{\circ}$ chamada conjunta EU-BR, acordo \#777067 (NECOS - Novel Enablers for Cloud Slicing). Este estudo foi também parcialmente financiado pela CAPES e CNPq. 


\section{Referências}

Clayman, S. (2017). Network slicing supported by dynamic vim instantatiation.

Clayman, S., Tusa, F., and Galis, A. (2018). Extending slices into data centers: the vim on-demand model. In 20189 th International Conference on the Network of the Future (NOF), pages 31-38. IEEE.

Dräxler, S., Karl, H., Peuster, M., Kouchaksaraei, H. R., Bredel, M., Lessmann, J., Soenen, T., Tavernier, W., Mendel-Brin, S., and Xilouris, G. (2017). Sonata: Service programming and orchestration for virtualized software networks. In 2017 IEEE International Conference on Communications Workshops (ICC Workshops), pages 973978. IEEE.

Freitas, L. A., Braga, V. G., Corrêa, S. L., Mamatas, L., Rothenberg, C. E., Clayman, S., and Cardoso, K. V. (2018). Slicing and allocation of transformable resources for the deployment of multiple virtualized infrastructure managers (vims). In 2018 4th IEEE Conference on Network Softwarization and Workshops (NetSoft), pages 424432. IEEE.

Han, B., Gopalakrishnan, V., Ji, L., and Lee, S. (2015). Network function virtualization: Challenges and opportunities for innovations. IEEE Communications Magazine, 53(2):90-97.

Kim, J., Kim, D., and Choi, S. (2017). 3gpp sa2 architecture and functions for $5 \mathrm{~g}$ mobile communication system. ICT Express, 3(1):1-8.

Mademann, F. (2017). System architecture milestone of $5 \mathrm{~g}$ phase 1 is achieved. Online verfügbar unter: http://www.3gpp.org/NEWS-EVENTS/3GPP-NEWS/1930SYS_ARCHITECTURE, letzter Zugriffam, 1:2018.

Ordonez-Lucena, J., Ameigeiras, P., Lopez, D., Ramos-Munoz, J. J., Lorca, J., and Folgueira, J. (2017). Network slicing for $5 \mathrm{~g}$ with $\mathrm{sdn} / \mathrm{nfv}$ : concepts, architectures and challenges. arXiv preprint arXiv:1703.04676.

Rost, P., Mannweiler, C., Michalopoulos, D. S., Sartori, C., Sciancalepore, V., Sastry, N., Holland, O., Tayade, S., Han, B., Bega, D., et al. (2017). Network slicing to enable scalability and flexibility in $5 \mathrm{~g}$ mobile networks. IEEE Communications magazine, 55(5):72-79.

Silva, F. S. D., Lemos, M. O., Medeiros, A., Neto, A. V., Pasquini, R., Moura, D., Rothenberg, C., Mamatas, L., Correa, S. L., Cardoso, K. V., et al. (2018). Necos project: Towards lightweight slicing of cloud federated infrastructures. In 2018 4th IEEE Conference on Network Softwarization and Workshops (NetSoft), pages 406-414. IEEE. 


\section{Referências}

Clayman, S. (2017). Network slicing supported by dynamic vim instantatiation.

Clayman, S., Tusa, F., and Galis, A. (2018). Extending slices into data centers: the vim on-demand model. In 20189 th International Conference on the Network of the Future (NOF), pages 31-38. IEEE.

Dräxler, S., Karl, H., Peuster, M., Kouchaksaraei, H. R., Bredel, M., Lessmann, J., Soenen, T., Tavernier, W., Mendel-Brin, S., and Xilouris, G. (2017). Sonata: Service programming and orchestration for virtualized software networks. In 2017 IEEE International Conference on Communications Workshops (ICC Workshops), pages 973978. IEEE.

Freitas, L. A., Braga, V. G., Corrêa, S. L., Mamatas, L., Rothenberg, C. E., Clayman, S., and Cardoso, K. V. (2018). Slicing and allocation of transformable resources for the deployment of multiple virtualized infrastructure managers (vims). In 2018 4th IEEE Conference on Network Softwarization and Workshops (NetSoft), pages 424432. IEEE.

Han, B., Gopalakrishnan, V., Ji, L., and Lee, S. (2015). Network function virtualization: Challenges and opportunities for innovations. IEEE Communications Magazine, 53(2):90-97.

Kim, J., Kim, D., and Choi, S. (2017). 3gpp sa2 architecture and functions for $5 \mathrm{~g}$ mobile communication system. ICT Express, 3(1):1-8.

Mademann, F. (2017). System architecture milestone of $5 \mathrm{~g}$ phase 1 is achieved. Online verfügbar unter: http://www.3gpp.org/NEWS-EVENTS/3GPP-NEWS/1930SYS_ARCHITECTURE, letzter Zugriffam, 1:2018.

Ordonez-Lucena, J., Ameigeiras, P., Lopez, D., Ramos-Munoz, J. J., Lorca, J., and Folgueira, J. (2017). Network slicing for $5 \mathrm{~g}$ with $\mathrm{sdn} / \mathrm{nfv}$ : concepts, architectures and challenges. arXiv preprint arXiv:1703.04676.

Rost, P., Mannweiler, C., Michalopoulos, D. S., Sartori, C., Sciancalepore, V., Sastry, N., Holland, O., Tayade, S., Han, B., Bega, D., et al. (2017). Network slicing to enable scalability and flexibility in $5 \mathrm{~g}$ mobile networks. IEEE Communications magazine, 55(5):72-79.

Silva, F. S. D., Lemos, M. O., Medeiros, A., Neto, A. V., Pasquini, R., Moura, D., Rothenberg, C., Mamatas, L., Correa, S. L., Cardoso, K. V., et al. (2018). Necos project: Towards lightweight slicing of cloud federated infrastructures. In 2018 4th IEEE Conference on Network Softwarization and Workshops (NetSoft), pages 406-414. IEEE. 


\section{Referências}

Clayman, S. (2017). Network slicing supported by dynamic vim instantatiation.

Clayman, S., Tusa, F., and Galis, A. (2018). Extending slices into data centers: the vim on-demand model. In 20189 th International Conference on the Network of the Future (NOF), pages 31-38. IEEE.

Dräxler, S., Karl, H., Peuster, M., Kouchaksaraei, H. R., Bredel, M., Lessmann, J., Soenen, T., Tavernier, W., Mendel-Brin, S., and Xilouris, G. (2017). Sonata: Service programming and orchestration for virtualized software networks. In 2017 IEEE International Conference on Communications Workshops (ICC Workshops), pages 973978. IEEE.

Freitas, L. A., Braga, V. G., Corrêa, S. L., Mamatas, L., Rothenberg, C. E., Clayman, S., and Cardoso, K. V. (2018). Slicing and allocation of transformable resources for the deployment of multiple virtualized infrastructure managers (vims). In 2018 4th IEEE Conference on Network Softwarization and Workshops (NetSoft), pages 424432. IEEE.

Han, B., Gopalakrishnan, V., Ji, L., and Lee, S. (2015). Network function virtualization: Challenges and opportunities for innovations. IEEE Communications Magazine, 53(2):90-97.

Kim, J., Kim, D., and Choi, S. (2017). 3gpp sa2 architecture and functions for $5 \mathrm{~g}$ mobile communication system. ICT Express, 3(1):1-8.

Mademann, F. (2017). System architecture milestone of $5 \mathrm{~g}$ phase 1 is achieved. Online verfügbar unter: http://www.3gpp.org/NEWS-EVENTS/3GPP-NEWS/1930SYS_ARCHITECTURE, letzter Zugriffam, 1:2018.

Ordonez-Lucena, J., Ameigeiras, P., Lopez, D., Ramos-Munoz, J. J., Lorca, J., and Folgueira, J. (2017). Network slicing for $5 \mathrm{~g}$ with $\mathrm{sdn} / \mathrm{nfv}$ : concepts, architectures and challenges. arXiv preprint arXiv:1703.04676.

Rost, P., Mannweiler, C., Michalopoulos, D. S., Sartori, C., Sciancalepore, V., Sastry, N., Holland, O., Tayade, S., Han, B., Bega, D., et al. (2017). Network slicing to enable scalability and flexibility in $5 \mathrm{~g}$ mobile networks. IEEE Communications magazine, 55(5):72-79.

Silva, F. S. D., Lemos, M. O., Medeiros, A., Neto, A. V., Pasquini, R., Moura, D., Rothenberg, C., Mamatas, L., Correa, S. L., Cardoso, K. V., et al. (2018). Necos project: Towards lightweight slicing of cloud federated infrastructures. In 2018 4th IEEE Conference on Network Softwarization and Workshops (NetSoft), pages 406-414. IEEE. 


\section{Referências}

Clayman, S. (2017). Network slicing supported by dynamic vim instantatiation.

Clayman, S., Tusa, F., and Galis, A. (2018). Extending slices into data centers: the vim on-demand model. In 20189 th International Conference on the Network of the Future (NOF), pages 31-38. IEEE.

Dräxler, S., Karl, H., Peuster, M., Kouchaksaraei, H. R., Bredel, M., Lessmann, J., Soenen, T., Tavernier, W., Mendel-Brin, S., and Xilouris, G. (2017). Sonata: Service programming and orchestration for virtualized software networks. In 2017 IEEE International Conference on Communications Workshops (ICC Workshops), pages 973978. IEEE.

Freitas, L. A., Braga, V. G., Corrêa, S. L., Mamatas, L., Rothenberg, C. E., Clayman, S., and Cardoso, K. V. (2018). Slicing and allocation of transformable resources for the deployment of multiple virtualized infrastructure managers (vims). In 2018 4th IEEE Conference on Network Softwarization and Workshops (NetSoft), pages 424432. IEEE.

Han, B., Gopalakrishnan, V., Ji, L., and Lee, S. (2015). Network function virtualization: Challenges and opportunities for innovations. IEEE Communications Magazine, 53(2):90-97.

Kim, J., Kim, D., and Choi, S. (2017). 3gpp sa2 architecture and functions for $5 \mathrm{~g}$ mobile communication system. ICT Express, 3(1):1-8.

Mademann, F. (2017). System architecture milestone of $5 \mathrm{~g}$ phase 1 is achieved. Online verfügbar unter: http://www.3gpp.org/NEWS-EVENTS/3GPP-NEWS/1930SYS_ARCHITECTURE, letzter Zugriffam, 1:2018.

Ordonez-Lucena, J., Ameigeiras, P., Lopez, D., Ramos-Munoz, J. J., Lorca, J., and Folgueira, J. (2017). Network slicing for $5 \mathrm{~g}$ with $\mathrm{sdn} / \mathrm{nfv}$ : concepts, architectures and challenges. arXiv preprint arXiv:1703.04676.

Rost, P., Mannweiler, C., Michalopoulos, D. S., Sartori, C., Sciancalepore, V., Sastry, N., Holland, O., Tayade, S., Han, B., Bega, D., et al. (2017). Network slicing to enable scalability and flexibility in $5 \mathrm{~g}$ mobile networks. IEEE Communications magazine, 55(5):72-79.

Silva, F. S. D., Lemos, M. O., Medeiros, A., Neto, A. V., Pasquini, R., Moura, D., Rothenberg, C., Mamatas, L., Correa, S. L., Cardoso, K. V., et al. (2018). Necos project: Towards lightweight slicing of cloud federated infrastructures. In 2018 4th IEEE Conference on Network Softwarization and Workshops (NetSoft), pages 406-414. IEEE. 


\section{Referências}

Clayman, S. (2017). Network slicing supported by dynamic vim instantatiation.

Clayman, S., Tusa, F., and Galis, A. (2018). Extending slices into data centers: the vim on-demand model. In 20189 th International Conference on the Network of the Future (NOF), pages 31-38. IEEE.

Dräxler, S., Karl, H., Peuster, M., Kouchaksaraei, H. R., Bredel, M., Lessmann, J., Soenen, T., Tavernier, W., Mendel-Brin, S., and Xilouris, G. (2017). Sonata: Service programming and orchestration for virtualized software networks. In 2017 IEEE International Conference on Communications Workshops (ICC Workshops), pages 973978. IEEE.

Freitas, L. A., Braga, V. G., Corrêa, S. L., Mamatas, L., Rothenberg, C. E., Clayman, S., and Cardoso, K. V. (2018). Slicing and allocation of transformable resources for the deployment of multiple virtualized infrastructure managers (vims). In 2018 4th IEEE Conference on Network Softwarization and Workshops (NetSoft), pages 424432. IEEE.

Han, B., Gopalakrishnan, V., Ji, L., and Lee, S. (2015). Network function virtualization: Challenges and opportunities for innovations. IEEE Communications Magazine, 53(2):90-97.

Kim, J., Kim, D., and Choi, S. (2017). 3gpp sa2 architecture and functions for $5 \mathrm{~g}$ mobile communication system. ICT Express, 3(1):1-8.

Mademann, F. (2017). System architecture milestone of $5 \mathrm{~g}$ phase 1 is achieved. Online verfügbar unter: http://www.3gpp.org/NEWS-EVENTS/3GPP-NEWS/1930SYS_ARCHITECTURE, letzter Zugriffam, 1:2018.

Ordonez-Lucena, J., Ameigeiras, P., Lopez, D., Ramos-Munoz, J. J., Lorca, J., and Folgueira, J. (2017). Network slicing for $5 \mathrm{~g}$ with $\mathrm{sdn} / \mathrm{nfv}$ : concepts, architectures and challenges. arXiv preprint arXiv:1703.04676.

Rost, P., Mannweiler, C., Michalopoulos, D. S., Sartori, C., Sciancalepore, V., Sastry, N., Holland, O., Tayade, S., Han, B., Bega, D., et al. (2017). Network slicing to enable scalability and flexibility in $5 \mathrm{~g}$ mobile networks. IEEE Communications magazine, 55(5):72-79.

Silva, F. S. D., Lemos, M. O., Medeiros, A., Neto, A. V., Pasquini, R., Moura, D., Rothenberg, C., Mamatas, L., Correa, S. L., Cardoso, K. V., et al. (2018). Necos project: Towards lightweight slicing of cloud federated infrastructures. In 2018 4th IEEE Conference on Network Softwarization and Workshops (NetSoft), pages 406-414. IEEE. 


\section{Referências}

Clayman, S. (2017). Network slicing supported by dynamic vim instantatiation.

Clayman, S., Tusa, F., and Galis, A. (2018). Extending slices into data centers: the vim on-demand model. In 20189 th International Conference on the Network of the Future (NOF), pages 31-38. IEEE.

Dräxler, S., Karl, H., Peuster, M., Kouchaksaraei, H. R., Bredel, M., Lessmann, J., Soenen, T., Tavernier, W., Mendel-Brin, S., and Xilouris, G. (2017). Sonata: Service programming and orchestration for virtualized software networks. In 2017 IEEE International Conference on Communications Workshops (ICC Workshops), pages 973978. IEEE.

Freitas, L. A., Braga, V. G., Corrêa, S. L., Mamatas, L., Rothenberg, C. E., Clayman, S., and Cardoso, K. V. (2018). Slicing and allocation of transformable resources for the deployment of multiple virtualized infrastructure managers (vims). In 2018 4th IEEE Conference on Network Softwarization and Workshops (NetSoft), pages 424432. IEEE.

Han, B., Gopalakrishnan, V., Ji, L., and Lee, S. (2015). Network function virtualization: Challenges and opportunities for innovations. IEEE Communications Magazine, 53(2):90-97.

Kim, J., Kim, D., and Choi, S. (2017). 3gpp sa2 architecture and functions for $5 \mathrm{~g}$ mobile communication system. ICT Express, 3(1):1-8.

Mademann, F. (2017). System architecture milestone of $5 \mathrm{~g}$ phase 1 is achieved. Online verfügbar unter: http://www.3gpp.org/NEWS-EVENTS/3GPP-NEWS/1930SYS_ARCHITECTURE, letzter Zugriffam, 1:2018.

Ordonez-Lucena, J., Ameigeiras, P., Lopez, D., Ramos-Munoz, J. J., Lorca, J., and Folgueira, J. (2017). Network slicing for $5 \mathrm{~g}$ with $\mathrm{sdn} / \mathrm{nfv}$ : concepts, architectures and challenges. arXiv preprint arXiv:1703.04676.

Rost, P., Mannweiler, C., Michalopoulos, D. S., Sartori, C., Sciancalepore, V., Sastry, N., Holland, O., Tayade, S., Han, B., Bega, D., et al. (2017). Network slicing to enable scalability and flexibility in $5 \mathrm{~g}$ mobile networks. IEEE Communications magazine, 55(5):72-79.

Silva, F. S. D., Lemos, M. O., Medeiros, A., Neto, A. V., Pasquini, R., Moura, D., Rothenberg, C., Mamatas, L., Correa, S. L., Cardoso, K. V., et al. (2018). Necos project: Towards lightweight slicing of cloud federated infrastructures. In 2018 4th IEEE Conference on Network Softwarization and Workshops (NetSoft), pages 406-414. IEEE. 